\title{
Notas sobre la construcción de estados de arte en ciencias sociales*
}

\author{
Jaime Alberto Saldarriaga-Vélez"* \\ Profesor Universidad de Antioquia, Colombia
}

Resumen: el presente artículo tiene la pretensión de contribuir a la reflexión epistemológica y metodológica sobre la construcción de estados de arte en procesos investigativos en las ciencias sociales, en Colombia. Parte de la distinción entre estados de arte entendidos como «investigación sobre la investigación»y los estados de arte que se constituyen en antecedentes del objeto de investigación, los cuales se tejen en articulación directa con el planteamiento del problema y con las categorías teóricas en las que se fundamenta un ejercicio investigativo académico. Este texto se inscribe en el análisis epistemológico tanto desde las perspectivas de conocimiento, interés y estilos de trabajo en ciencias sociales como también desde perspectivas contemporáneas de conocimiento: la mirada deconstructiva (posestructuralista) y la hermenéutica crítica. Propone además algunos elementos metodológicos derivados de dicho análisis, la selección del material pertinente, según criterios epistemológicos, teóricos y metodológicos, así como una matriz para el análisis del material seleccionado con base en las categorías y subcategorías, tanto previas como emergentes. El producto final de este proceso constituye un conjunto de antecedentes del proyecto de investigación, sin el cual la propuesta investigativa pierde validez.

Palabras clave: estado de arte; antecedentes; metodología de investigación; ciencias sociales, hermenéutica (Eric Thesaurus y Tesauro de Ciencias Sociales de la Unesco).

* Este texto es el resultado de la revisión crítica del tema de estados de arte en la investigación en Ciencias Sociales en Colombia, realizada en el marco de la Línea de Educación y Pedagogía del Doctorado en Ciencias Sociales, Niñez y Juventud, Universidad de Manizales-Cinde (Manizales, Colombia), entre los meses de enero y mayo de 2017 .

*: Licenciado en Educación, Filosofía-Historia. Doctor en Ciencias Sociales, Niñez y Juventud (Universidad de Manizales, Cinde). Investigador educativo, Corporación Región (Medellín). Docente, Universidad de Antioquia. Correo electrónico: jaimesaldarriaga@hotmail.com 


\title{
Notes on the construction of States-of-the-Art in social sciences
}

\begin{abstract}
The paper has the pretension to contribute to the epistemological and methodological reflection on the construction of States of the Art in research processes in Social Sciences, in Colombia. Its starting point is the distinction between States of the Art understood as research on research, and the States of the Art that constitute antecedents of the object of research, which are woven in direct articulation with the approach of the problem and with the categories theories on which an academic research exercise is based. This text is part of the epistemological analysis, both from the perspective of knowledge and interest and work styles in social sciences, as well as from contemporary perspectives of knowledge such as the gaze deconstructive (post-structuralist) and critical hermeneutics. It proposes some methodological elements derived from this analysis. The final product of this process is a set of background of the research project, without which the research proposal loses its validity.
\end{abstract}

Keywords: State of the Art; Background; Research Methodology; Social Sciences; Hermeneutics.

\section{Notas sobre a construção de estados de arte em ciências sociais}

Resumo: o presente artigo tem a pretensão de contribuir à reflexão epistemológica e metodológica sobre a construção de estados de arte em processos investigativos em ciências sociais, na Colômbia. Tem como ponto de partida a distinção entre estados de arte entendidos como «investigação sobre a investigação», e os estados de arte que se constituem em antecedentes do objeto de investigação, que se tecem em articulação direta com a proposta do problema e com as categorias teóricas nas que se fundamenta um exercício investigativo acadêmico. Este texto inscreve-se na análise epistemológico tanto desde as perspectivas de conhecimento, interesse e estilos de trabalho em ciências sociais, como também desde perspectivas contemporâneas de conhecimento: a mirada deconstructiva (pós-estructuralista) e a hermenêutica crítica. Propõe ademais alguns elementos metodológicos derivados de dito análise. O produto final deste processo é um conjunto de antecedentes do projeto de pesquisa, sem o qual a proposta de pesquisa perde sua validade.

Palavras chave: Estados de arte; Antecedentes; Metodologia da investigação; Ciências sociais; Hermenêutica. 


\section{Necesarias distinciones}

En Colombia el estado de arte en las ciencias sociales ha sido entendido tanto como investigación sobre la investigación (desarrollo de un campo investigativo) como investigación sobre lo investigado (sobre un objeto específico o «fenómeno social»). Igualmente, ha sido tomado también como una modalidad de investigación y como una estrategia metodológica (Guevara, 2016, p. 166), lo que nos muestra la necesidad de profundizar y avanzar en precisiones en cuanto a cómo entenderlo y desarrollarlo desde una u otra comprensión. Esto se debe en gran medida a que la presencia del estado de arte en el mundo investigativo colombiano, tanto en los centros de investigación como en los procesos de formación universitaria en pregrado y posgrado, es relativamente reciente, de finales de los años 80, como lo afirman los investigadores Absalón Jiménez y Gloria Calvo (Calvo, 1992; Jiménez, 2006). Antes de esa época su principal interés estuvo en el campo de la investigación historiográfica y económica (historiografía documental) o promovido por organismos gubernamentales y de cooperación (ONU, Unesco, Cepal, PNUD, entre otros) y ONG nacionales con la finalidad de sustentar apuestas políticas y de desarrollo social:

Se pretendía, inicialmente, establecer bajo su denominación lo que se sabía sobre una temática con la intención de fundamentar políticas y alternativas de acción para el desarrollo social. Cuando se trató de hacer estado del arte se quiso, inicialmente, analizar qué se sabía sobre cada una de las temáticas en toda la región de América Latina, es decir, los estados del arte nacieron con la pretensión de hacer un balance de la investigación en la región. (Guevara, 2016, p. 168)

Por tanto, los estados de arte (que podrían mejor denominarse, en aras de avanzar en la claridad metodológica, estados de la cuestión) comenzaron a realizarse en el país, no con una preocupación teórica, sino con la finalidad expresa de darle soporte técnico a los procesos de intervención social de dichos organismos. Es decir, su interés no era propiamente la investigación sino las políticas, programas y proyectos de desarrollo social; esto es, una investigación sobre objetos sociales específicos para ajustar o producir nuevos proyectos, así como incidir en políticas económicas y sociales desde una fundamentación científica, así como formular recomendaciones desde un soporte empírico convalidado académicamente. Sin embargo, es en los años noventa que en Colombia comenzaron a desarrollarse estudios documentales cuyo objeto no era el mundo social o la «realidad social» (como se suele nombrar), sino las investigaciones cuya pretensión era dar cuenta de un campo de conocimiento y producir teoría, es decir, hacer investigación sobre la investigación: 
En esa época, la autora (Gloria Calvo) abrió la discusión sobre el estado del arte y lo definió como una revisión de lo que se había producido sobre un tema; su producción habría de examinarse en los textos, es decir, no se trataba de mirar los documentos para fundamentar nuevas propuestas, sino que las propuestas de investigación y sus productos se convertían en un nuevo campo de investigación. (Guevara, 2016, p. 168)

Así, este tipo de investigación, aunque su objeto directo no fue inicialmente el mundo social, se ocupó tanto de los avances en la construcción epistemológica y teórica sobre un objeto (investigación sobre lo investigado), como del modo (metodológico) para investigarlo, con la pretensión de hacer un balance sobre los distintos desarrollos (investigación sobre la investigación) y de encontrar las directrices para profundizar o reorientar un campo de conocimiento.

En este sentido, los estados de arte se han constituido en un antecedente necesario para su abordaje investigativo, ya sea configurando un campo (inductivo) o construyendo y resignificando un objeto de investigación (deductivo), en tanto permiten aproximarse a un campo o un objeto, desde las distintas tradiciones metodológicas y perspectivas epistemológicas, teóricas y políticas:

Los estados de arte en ciencias sociales, y los producidos en cualquier tipo de investigación, representan el primer paso de acercamiento y apropiación de la realidad como tal, pero, ante todo, esta propuesta metodológica se encuentra mediada por los textos y los acumulados que de las ciencias sociales ellos contienen. (Jiménez, 2006, p. 31)

Asimismo, los estados de arte son ejercicios inductivos si se parte de los textos a la emergencia de categorías; deductivos, si se parte de categorías iniciales y se les hace rastreo a sus desarrollos; e inductivos/deductivos o deductivos/inductivos si, partiendo de las categorías o de los textos, se va y vuelve en doble vía. La elección de uno u otro camino depende en gran medida de las perspectivas epistemológicas, pero también de la finalidad con la que se construyan los estados de arte. De estos dos criterios, el epistemológico y el pragmático, va a depender el camino que se tome, esto es, el método de elaboración de un estado de arte.

Por ejemplo, los estados de arte que se construyen para las investigaciones académicas (pregrado, posgrado) tienen la finalidad de afianzar la comprensión de un objeto y profundizar en el planteamiento del problema de investigación, de ahí su punto de partida y su razón de ser; por consiguiente, recaban sobre lo investigado en el objeto específico, analizan las perspectivas epistemológicas, los enfoques temáticos y las tradiciones metodológicas desde las cuales 
se han construido. También facilitan la fundamentación de las categorías, sea que estas hayan sido predefinidas por el investigador o que emerjan de la realización del estado de arte, ya porque se desea continuar una tradición de lo previamente investigado o porque se busque identificar vacíos teóricos para lo que habrá que explorar e instalar categorías emergentes. Es decir, el resultado de un estado de arte es un balance sobre el conjunto de investigaciones revisadas, en el cual se señalan los avances, vacíos conceptuales y temáticos identificados, y las tendencias metodológicas, teniendo como referente de análisis las perspectivas epistemológicas y políticas desde las que se aborda un campo u objeto. De estas últimas se ocupa el siguiente apartado.

\section{Posturas epistemológicas y políticas de los estados de arte}

En los estados de arte (no solo en su elaboración sino también en los análisis y en los balances del estado actual del campo o del objeto) es importante abordar las posturas epistemológicas y políticas en las que están inscritos. Al respecto, Páramo (2008) afirma:

Se entiende por postura epistemológica o paradigma el conjunto de suposiciones de carácter filosófico de las que nos valemos para aproximarnos a la búsqueda del conocimiento, la noción que compartimos de realidad y de verdad, y el papel que cumple el investigador en esta búsqueda de conocimiento, al igual que la manera como asumimos al sujeto estudiado. (p. 21)

En este mismo sentido, Jiménez (2006) expresa:

Cuando hablamos de estado del arte para el abordaje de un problema o un tema en cualquiera de las ciencias sociales, estamos hablando de la necesidad hermenéutica de remitirnos a textos que a su vez son expresiones de desarrollos investigativos, dados desde diversas percepciones de las ciencias sociales y escuelas de pensamiento - el funcionalismo, el marxismo o el estructuralismo-, tarea emprendida y cuyo objetivo final es el conocimiento y la apropiación de la realidad social para luego disertarla y problematizarla. (p. 32 )

Así, las diferentes nociones de ciencias, de realidad, de verdad y de investigación que se derivan de cada perspectiva, se constituyen en el marco epistemológico desde el cual se configuran los distintos estados de arte. Siguiendo a Habermas (1982) y a Vasco (1989), se retoman las perspectivas epistemológicas que comportan intereses intra-y extrateóricos: «predecir y controlar, para el interés técnico; ubicar y orientar (la praxis social y personal dentro de la historia), para el interés práctico, y develar y romper, liberar, para el interés emancipatorio» (Vasco, 1989, p. 28). Además de estos intereses, ya reconocidos y aplicados en la investigación, los 
desarrollos contemporáneos de las ciencias sociales muestran perspectivas epistemológicas e intereses emergentes como el interés deconstructivo de relaciones de poder, de saberes, de verdades, de ciencias y disciplinas (Derrida, Foucault, Deleuze, Serres, entre otros pensadores de la sociedad), que se diferencian de las perspectivas señaladas por la Escuela de Frankfurt.

Por todo esto, el análisis de las perspectivas en los estados de arte, no solo permite clasificarlos desde la posición del investigador sino, también profundizar en las tendencias epistemológicas y políticas del campo y del objeto específico de las distintas investigaciones; tal como sostiene Guevara (2016): «La intención es validar la idea de que la postura epistemológica se convierte en columna vertebral del desarrollo de un estado de arte» (p. 166). En este sentido, un estado de arte diseñado desde el interés técnico, construido desde la perspectiva tecnofuncional (ciencia positiva) de las ciencias sociales, privilegia el plano descriptivo de los materiales, cuantificando tendencias para ser generalizadas, más allá de las particularidades que muestra la contextualización: «En relación con el estado de arte se verifican y miden variables para describir y caracterizar los documentos» (Guevara, 2016, p. 172).

El producto final muestra cuadros de tendencias, elaborados desde la estadística descriptiva, como instrumentos básicos y necesarios para el tratamiento de los datos, privilegiando el análisis cuantitativo de los resultados obtenidos en las distintas investigaciones. Se parte del supuesto de una realidad objetiva que es posible determinar en sus regularidades para prever su desarrollo e intervenirlo. Vale decir que este modo de hacer los estados de arte se constituye en instrumento de recopilación y acumulación de evidencias sobre un objeto; es decir, los estados de arte se convierten en un instrumento importante para consolidar tendencias en descubrimientos y hallazgos, como dato positivo.

La aplicación de técnicas cuantitativas estadísticas puede ser de utilidad para algunos estados de arte en investigaciones cualitativas, en cuanto sirva para evidenciar tendencias en una base documental; no obstante, por sí misma resulta insuficiente para la comprensión de acontecimientos y actores sociales, como un todo-con-sentido.

Desde la perspectiva hermenéutica se privilegia la interpretación de los textos, utilizando herramientas de análisis de discurso, referenciando las diferentes narrativas, su intencionalidad, los lenguajes y marcos culturales en que se construyen las investigaciones, así como sus desarrollos históricos. Parte del supuesto epistemológico de que el mundo y su conocimiento (y en él las ciencias) son construcciones de sentido, estructuras de lenguaje, juegos de lenguaje. A partir de esta perspectiva se entiende que los estados de arte son un ejercicio hermenéutico, en una interacción permanente entre los autores, textos, contextos, en el cual desaparece la pretensión de objetividad. Es un intercambio de comprensiones como interacción social: la del 
texto, la de su autor, la de la comunidad científica, la del mundo cultural y simbólico en el que se inscribe una investigación. De acuerdo a Vasco:

El estilo ya no es predominantemente analítico, en el sentido de tratar de desbaratar en un sistema y dejarlo separado para poderlo ver claro. La historia y la hermenéutica tratan precisamente de reconstruir todas las piezas aisladas que aparecen en las diversas interpretaciones de los hechos, en los diversos textos, en las diversas versiones, en los diversos hallazgos arqueológicos, literarios, lingüísticos, para recapturar un «todo-con-sentido». (Vasco, 1989, p. 20)

Se trata, entonces, de un ejercicio reflexivo comprensivo al que le interesa encontrar, no la «realidad» ni lo acontecido, ni los consensos en los resultados de las distintas investigaciones que puedan presentarse - como ciencia y verdad - sino las diferentes lecturas; y no solo del hecho sino de las diversas comprensiones y narrativas que, sobre un acontecimiento social, se construyen, tanto por los investigadores como por los propios agentes sociales (presentes a través de testimonios, relatos y otras fuentes, consignados en los textos escritos).

Haciendo eco de esta perspectiva hermenéutica de los estados de arte, Guevara (2016) la propone como: «un tipo de investigación documental eminentemente interpretativa, cuyo diseño es cíclico y está formado por la descripción-interpretación-elaboración o construcción teórica» (p. 174). El proceso de construcción del estado de arte desde esta perspectiva se da como un «círculo hermenéutico», que va del dato (el texto literal) al análisis del discurso, desde sus diversos enfoques, para luego aproximarse a una síntesis interpretativa sobre sentido histórico y cultural de un acontecimiento social, en proceso cíclico continuo.

En la perspectiva emancipatoria socio-crítica (liberación), los estados de arte incorporan de manera explícita una posición crítica frente a las posturas epistemológicas y políticas de las investigaciones y de los investigadores, sobre el presupuesto de un cuestionamiento de la neutralidad política, valorativa e investigativa, tanto del investigador, del estado de arte producido, como de la selección y análisis de las investigaciones tomadas para construirlo. De esta manera, valida las investigaciones especialmente por su postura crítico-social, por ser una investigación militante y comprometida con los procesos emancipatorios: «El paradigma de la teoría crítica concibe la realidad cuando el sujeto se reconoce activamente, cuando participa con otros grupos o comunidades en compromiso hacia la acción emancipatoria» (Guevara, 2016, p. 174). Por tanto, los estados de arte, desde esta perspectiva, no se conciben como simples ejercicios académicos sino como parte de la praxis política del investigador, que no se entiende ya como un investigador individual sino como un actor social generador de conocimiento crítico. En este sentido, su producción se vincula a procesos sociales como parte de un 
colectivo:

Desde el punto de vista ontológico, el estado de arte plantea que el investigador construya puentes entre el lector y el texto, el texto y su productor, el contexto histórico y el presente, y una circunstancia social en particular y otra que impliquen la transformación hacia una práctica democrática. (Guevara, 2016, p. 174)

Para esta perspectiva, el hecho de que la elaboración de estados de arte se construya sobre la lectura crítico-social de los textos lleva a los investigadores críticos a recurrir a la hermenéutica, a la cual le aportan el criterio político para la realización de análisis del discurso, fijando su mirada en los proceso históricos y sociales, en sus actores, en las posturas ideológicas de los investigadores de un acontecimiento o hecho social y en las contradicciones entre las posturas de estos en su interpretación. El recurso de investigadores sociocríticos a la hermenéutica ha configurado una vertiente metodológica que hoy se puede denominar hermenéutica crítica, la cual puede reconocerse en autores como Boaventura de Souza Santos, Óscar Mejía-Quintana, Jesús Martín-Barbero, y distintos investigadores inscritos en el pensamiento decolonial, los estudios culturales, las pedagogías críticas, entre otras corrientes que han articulado los estudios del lenguaje y la cultura a la investigación sociocrítica.

Los desarrollos contemporáneos de las ciencias sociales, de la mano de los estudios lingüísticos, han desarrollado una nueva reflexión que toma distancia de las perspectivas señaladas por Habermas, configurándose un pensamiento de la deconstrucción; esta llamado genéricamente como posestructuralismo y al que se asocian autores como Michel Foucault, Jacques Derrida, Gilles Deleuze, Michel Serres, entre otros. Esta perspectiva se ha fijado de manera particular en los discursos constitutivos del conocimiento moderno occidental a partir de la tríada poder/saber/verdad. A diferencia del pensamiento sociocrítico, toma distancia de la apuesta utópica occidental (tanto liberal como marxista) y de su concepción de la realidad (entendida ya no como un dato empírico sino como estructura de lenguaje, como símbolo), haciendo de la deconstrucción de verdades, poderes y dispositivos sociales de configuración de subjetividades su objeto de investigación.

Metodológicamente, los investigadores deconstructivos han utilizado el análisis de formaciones discursivas y de las relaciones de poder/saber/verdad (Arqueología de saber, Foucault, 1979), el análisis estructural de contenido (Hiernaux, Saldarriaga, Suárez) y un especial instrumento de gran utilidad para la construcción de estados de arte como lo es el archivo, entendido «no como la suma de todos los textos que una cultura ha guardado», ni como las instituciones que los conservan, sino como «la ley de lo que pudo ser dicho, el sistema que rige la 
aparición de los enunciados como acontecimientos singulares» (Foucault, 1979, p. 219). De esta manera, los estados de arte, en su configuración documental como corpus investigativo, han de ser sometidos también a la crítica de los modos de aparición y reconocimiento de dichos documentos. Esto se constituye en un modo deconstructivo de investigación sobre la investigación.

Así pues, los estados de arte se constituyen también en un campo de conocimiento y de disputa, mediados por las perspectivas epistemológicas y políticas de estilos de trabajo, de conocimiento e interés en las ciencias sociales. En este sentido, los estudios genealógicos que se derivan de los estados de arte, permiten analizar los dispositivos de poder que han configurado instituciones disciplinarias y de control, lo que posibilita leer las investigaciones a partir de los discursos de disciplinamiento y control, de configuración de subjetividades que las mismas investigaciones registran o agencian. En Colombia, especialmente en los campos de la educación, la pedagogía y la infancia, es necesario resaltar los trabajos del grupo de la Historia de la Práctica Pedagógica, en particular de los investigadores Olga Lucía Zuluaga, Javier Sáenz, Óscar Saldarriaga, Jesús Alberto Echeverri, entro otros.

\section{Algunos elementos metodológicos en la construcción de estados de arte en trabajos de investigación académicos o «antecedentes»}

En la literatura sobre los estados de arte en ciencias sociales existen coincidencias que es necesario señalar como referente inicial en cuanto a su construcción metodológica; por ejemplo, que los estados de arte son propiamente un ejercicio hermenéutico. Por consiguiente, van mucho más allá de la identificación y recolección descriptiva documental y de otras fuentes. No existe un único método para la realización de estados de arte (Guevara, 2016), por tanto, tienen como punto de partida el planteamiento del problema, su contextualización, las categorías iniciales, la revisión bibliográfica de entrada. Ello implica que

es una recopilación crítica de diversos tipos de texto de un área o disciplina que, de manera escrita, formaliza el proceso cognitivo de una investigación a través de la lectura de la bibliografía hallada durante la indagación del problema, los temas y los contextos. (Londoño, Maldonado, \& Calderón, 2014, p. 7)

Además, la elaboración de los estados de arte comporta al menos dos componentes fundamentales: un componente heurístico o de exploración de los documentos a ser incorporados en un determinado estado de arte y un componente interpretativo articulado al primero y en interrelación constante. El componente heurístico se toma como primer momento y se ocupa principalmente de la exploración de materiales textuales para su posterior interpretación. Este 
primer momento es llamado por algunos investigadores, como la investigadora Gloria Calvo (1992), de contextualización y consiste en una delimitación inicial de la búsqueda de material derivado del planteamiento inicial del problema. De ello depende que esta búsqueda inicial no se desborde, y que se esté ajustando constantemente a criterios de pertinencia y relevancia. Por esta razón, la fase heurística o exploratoria requiere ser hecha con sentido, teniendo como referentes los desarrollos iniciales del planteamiento del problema y de la pregunta de investigación, así como las categorías iniciales, que permitan al investigador ir apropiándose y profundizando en el campo y en el objeto, en una triangulación entre el planteamiento del problema, el conocimiento acumulado sobre el objeto y los desarrollos del marco conceptual (las categorías iniciales) para abrir la posibilidad de otras comprensiones.

Para facilitar la recolección de información, su ordenamiento e interpretación, se requiere del diseño y uso de distintas fichas de registro (resumen analítico especializado - RAE-), de fichas bibliográficas, de un sistema de codificación de estas y de una matriz inicial de categorías y subcategorías derivadas del planteamiento inicial del problema (abierto a categorías emergentes). Las fichas deben adecuarse al tipo de material dependiendo de su origen: materiales académicos sobre el problema, provenientes de proyectos de investigación, materiales provenientes de programas o proyectos (Barbosa, Barbosa, \& Rodríguez, 2013), materiales provenientes de experiencias sociales, entre otros posibles.

Los materiales buscados deben ser inicialmente clasificados y seleccionados, definiendo su pertinencia y calidad, para posteriormente pasar a ser fichados. Fichar todo lo que se encuentra sin valorar su pertinencia e importancia antes de dicha clasificación y selección, puede llevar a una pérdida de tiempo innecesaria, a una pérdida de rumbo, así como a un desgaste emocional del investigador, apenas al comienzo de su trabajo. Una vez seleccionado y fichado el material se procede a su codificación, para lo cual se recomienda construir una matriz de categorías y subcategorías y proceder a organizar los registros ya seleccionados, ubicándolos en dicha matriz. Este paso se puede considerar ya parte del proceso hermenéutico.

Finalmente, se procede al análisis del material codificado, a partir de la matriz, estableciendo relaciones entre los elementos: continuidad, ruptura, dependencia, autonomía, complementariedad, regularidad, vacíos, abundancia, tendencias en enfoque, categorías o subcategorías emergentes, entre otros rasgos que pueden ser considerados para el análisis.

\section{Conclusión}

Los estados de arte se ocupan del abordaje crítico de las investigaciones que anteceden el objeto (en construcción), el cual debe posibilitarle al investigador hacer un balance inicial de 
los desarrollos teóricos y metodológicos de este, y su articulación con el campo en el que se inscribe. A partir de dicho balance, el investigador deberá tomar decisiones en cuanto a la pertinencia y relevancia del problema planteado y esto le permitirá identificar aspectos poco abordados y que exigen ser desarrollados. Igualmente, le posibilitará enriquecer el planteamiento inicial, encontrarse con los desarrollos teóricos y con los enfoques metodológicos construidos por otros investigadores. Para ello, se requerirá de instrumentos para la recolección, la clasificación, el ordenamiento, la jerarquización del material recolectado (material principal/ material secundario) y la selección del material pertinente (según criterios epistemológicos, teóricos y metodológicos), así como de una matriz para el análisis del material seleccionado con base en las categorías y subcategorías, tanto previas como emergentes. El producto (escrito) de este proceso constituye un conjunto de antecedentes del proyecto de investigación, sin el cual la propuesta investigativa pierde validez.

\section{Referencias}

Barbosa, J. W., Barbosa, J. C., \& Rodríguez, M. (2013). Revisión y análisis documental para estado del arte: una propuesta metodológica desde el contexto de la sistematización de experiencias educativas. Revista Investigación Bibliotecológica, 27(61), 83-105.

Calvo, G. (1992). Análisis de la investigación en la formación de investigadores. Bogotá, D. C.: Universidad de La Sabana.

Foucault, M. (1979). Arqueología del saber. Ciudad de México: Siglo XXI.

Guevara, R. (2016). El estado del arte en la investigación: ¿análisis de los conocimientos acumulados o indagación por nuevos sentidos? Revista Folios, (44), 165-179.

Habermas, J. (1982). Conocimiento e interés. Madrid: Taurus.

Jiménez, A. (2006). La práctica investigativa en Ciencias Sociales. En R. Ávila, A. Jiménez, \& A. Torres (Autores), La práctica investigativa en ciencias sociales (pp. 28-42). Bogotá, D. C.: Departamento de Ciencias Sociales, Universidad Pedagógica Nacional.

Londoño, O., Maldonado, L., \& Calderón, L. (2014). Guía para construir estados de arte. Bogotá,

D. C.: International Corporation of Networks of Knowledge.

Páramo, P. (2008). La investigación en las ciencias sociales: técnicas de recolección de información.

Bogotá, D. C.: Universidad Piloto de Colombia.

Vasco, C. (1989). Tres estilos de trabajo en Ciencias Sociales. Bogotá, D. C.: Cinep. 